\title{
THE IMPACT OF A 10-YEAR MUSCULOSKELETAL ULTRASOUND TRAINING PROGRAM ON FORMER FELLOWS CLINICAL PRACTICE
}

\author{
Rafael Mendonça da Silva Chakr ${ }^{1, \star}$ \\ 1.Universidade Federal do Rio Grande do Sul, Porto Alegre (RS), Brazil. \\ *Corresponding author: rchakr@hcpa.edu.br
}

\section{BACKGROUND}

Musculoskeletal ultrasound (MSKUS) training has become part of the rheumatology fellowship program worldwide. Our goal is to report the impact of a 10-year structured MSKUS training program on former fellows practice.

\section{METHODS}

In November 2020, all former fellows of a university hospital trained by the same professor between 2011 and 2020 were invited to participate in an anonymous survey, consisting of multiple-choice questions and $0-10$ Likert scales ( $0=$ nothing; $10=$ very much). During the last year of the training program, all fellows were engaged in regular theoretical and practical adult MSKUS activities twice a week. The MSKUS teaching curriculum consisted of image optimization, ultrasound physics, identification of normal anatomy and pathologies with disease-specific findings via hands-on scanning of joints, and hands-on guided procedures/injections. Trainees were also encouraged to participate in the Brazilian Society of Rheumatology MSKUS training courses during their fellowship. For proportions comparisons, Fisher's exact test was used.

\section{RESULTS}

Out of the 25 rheumatologists invited, 20 responded to the questionnaire. In total, $55 \%$ had less than 5 years of post-fellowship practice, $90 \%$ dedicated more than half of their working hours to patient care and $10 \%$ were performing MSKUS regularly. For $55 \%$ of the respondents, there was at least one rheumatologist performing MSKUS in the same city. When asked how much MSKUS training during fellowship had contributed to improving their clinical practice, $75 \%$ answered $\geq 7$ on a $0-10$ scale. Clinical practice impact was neither associated with years after completing fellowship ( $p=1.000$ ) nor with the presence of another rheumatologist performing MSKUS locally ( $p=0.617$ ). Overall, $85 \%$ of rheumatologists agreed $\geq 7$ on a $0-10$ scale with the sentence "if the access to ultrasound machines was facilitated, I believe MSKUS could increase my earnings". That percentage was 60\% among more experienced rheumatologists that lived in the same city as another rheumatologist performing MSKUS. Potential reimbursement increment was neither associated with years after completing fellowship ( $p=0.537$ ) nor with the presence of a rheumatologist performing MSKUS in the same city ( $p=0.218$ ). For $60 \%$ of former fellows, MSKUS training period should be increased from one to two years during fellowship.

\section{CONCLUSION}

Within 10 years of observation, in former fellows' perception, a curriculum-based MSKUS training program during rheumatology fellowship seems to improve subsequent patient care and could contribute to increasing earnings if ultrasound machine access was facilitated.

\section{KEYWORDS}

Ultrasound, Training, Fellowship. 